\title{
Brittle fracture in polycrystalline microstructures with the extended finite element method
}

\author{
N. Sukumar ${ }^{1,2, *, \dagger}$, D. J. Srolovitz ${ }^{2,3}$, T. J. Baker ${ }^{3}$ and J.-H. Prévost ${ }^{2,4}$ \\ ${ }^{1}$ Department of Civil and Environmental Engineering, University of California, Davis, CA 95616, U.S.A. \\ ${ }_{2}^{2}$ Princeton Materials Institute, Bowen Hall, Princeton University, NJ 08544, U.S.A. \\ ${ }^{3}$ Department of Mechanical and Aerospace Engineering, Princeton University, NJ 08544, U.S.A. \\ ${ }^{4}$ Department of Civil and Environmental Engineering, Princeton University, NJ 08544, U.S.A.
}

\begin{abstract}
SUMMARY
A two-dimensional numerical model of microstructural effects in brittle fracture is presented, with an aim towards the understanding of toughening mechanisms in polycrystalline materials such as ceramics. Quasi-static crack propagation is modelled using the extended finite element method (X-FEM) and microstructures are simulated within the framework of the Potts model for grain growth. In the X-FEM, a discontinuous function and the two-dimensional asymptotic crack-tip displacement fields are added to the finite element approximation to account for the crack using the notion of partition of unity. This enables the domain to be modelled by finite elements with no explicit meshing of the crack surfaces. Hence, crack propagation can be simulated without any user-intervention or the need to remesh as the crack advances. The microstructural calculations are carried out on a regular lattice using a kinetic Monte Carlo algorithm for grain growth. We present a novel constrained Delaunay triangulation algorithm with grain boundary smoothing to create a finite element mesh of the microstructure. The fracture properties of the microstructure are characterized by assuming that the critical fracture energy of the grain boundary $\left(G_{\mathrm{c}}^{\mathrm{gb}}\right)$ is different from that of the grain interior $\left(G_{\mathrm{c}}^{\mathrm{i}}\right)$. Numerical crack propagation simulations for varying toughness ratios $G_{\mathrm{c}}^{\mathrm{gb}} / G_{\mathrm{c}}^{\mathrm{i}}$ are presented, to study the transition from the intergranular to the transgranular mode of crack growth. This study has demonstrated the capability of modelling crack propagation through a material microstructure within a finite element framework, which opens-up exciting possibilities for the fracture analysis of functionally graded material systems. Copyright (C) 2003 John Wiley \& Sons, Ltd.
\end{abstract}

KEY WORDS: polycrystalline microstructure; grain boundary; strong discontinuity; two-dimensional crack propagation; Potts grain growth model; Delaunay triangulation; meshing microstructures; partition of unity; extended finite element method

\footnotetext{
*Correspondence to: N. Sukumar, Department of Civil and Environmental Engineering, University of California, Davis, CA 95616, U.S.A.

†E-mail: nsukumar@ucdavis.edu
}

Contract/grant sponsor: Idaho National Engineering Laboratory; contract/grant number: KOO-182412

Contract/grant sponsor: National Science Foundation; contract/grant number: NSF-9988788

Received 31 August 2001

Revised 31 January 2002

Copyright (c) 2003 John Wiley \& Sons, Ltd.

Accepted 10 June 2002 


\section{INTRODUCTION}

Understanding deformation and failure mechanisms in brittle polycrystalline materials such as ceramics is critical for improvements in the development and application of advanced structural materials. The material microstructure plays a pivotal role in dictating the modes of fracture and failure, and the macroscopic response of real materials. The grain morphology, elastic modulus, and the toughness of the individual microstructural constituents and interfaces are key parameters that control the failure mechanisms in polycrystalline materials. Concepts such as grain boundary design and control and grain boundary engineering to improve the fracture resistance of polycrystalline materials are well-recognized [1-3]. Quasi-static crack propagation through a material microstructure depends on the mechanical state in the vicinity of the crack-tip, and hence local differences in toughness (grain interior versus grain boundaries) significantly influence the crack path. In light of the above, it is clear that any numerical fracture model that is able to model crack propagation by incorporating these microstructural features has the potential to describe toughening mechanisms in polycrystals and provide a framework for microstructural design.

Brittle fracture simulations in disordered (heterogeneous) materials using spring networks has received wide attention since the late 1980s [4-6]. A detailed study on spring-networks and finite element methods for crack propagation simulations was conducted by Jagota and Bennison $[7,8]$, who pointed out the dilemma associated with spring networks - regular meshes can model uniform strain but show strong anisotropy in crack propagation whereas random networks cannot in general represent uniform strain. Schlangen and Garboczi [9] considered the appropriate selection of cross-sectional area and moment of inertia for beam elements in a random lattice to simulate a homogeneous medium with showed no mesh dependency. An elegant solution and partial resolution to the above shortcomings was provided by Bolander and Saito [10] who used rigid-body spring networks to model brittle fracture in homogeneous isotropic materials such as cement and concrete. They proposed a model for random networks based on the Voronoi tessellation which was able to produce homogeneous deformation on uniform straining and showed little bias towards crack propagation directions.

Lattice spring models have been used to study brittle fracture and damage in polycrystalline materials. Yang and co-workers [11] used the Potts grain growth model [12-16] to generate a polycrystalline microstructure which was mapped onto a triangular lattice. The mechanics of this structure was represented by a spring network on the lattice, where a spring fails if the stored elastic energy in the spring exceeded a critical value. In Reference [11], the transition from intergranular (growth along the grain boundary) to transgranular (growth in the grain interior) fracture with increasing grain boundary toughness was observed. In Reference $[17,18]$, the effects of thermal-mismatch on microcracking was studied. Holm [19] considered surface formation energies to study intergranular fracture in polycrystals; the influence of low/high-angle grain boundaries and grain boundary microcracking on the fracture path was investigated. Kim and co-workers [20] analysed two-dimensional crack propagation through a polycrystal as a function of the grain boundary toughness, focusing on the competition between intergranular and transgranular mode of crack propagation.

An alternative approach to modelling fracture phenomena for arbitrary microstructure is the use of cohesive surfaces within a finite element formulation. Zhai and Zhou [21] proposed a micromechanical model in which the cohesive surface formulation of $\mathrm{Xu}$ and Needleman [22] is used to study failure modes in composite microstructures, whereas in Reference [23], 
intergranular microcrack initiation and evolution is assessed in ceramic microstructures under dynamic loading conditions. The cohesive surface formulation is a phenomenological framework in which the fracture characteristics of the material are embedded in a cohesive surface traction-displacement relation. Using this approach, an inherent length scale is introduced into the model, and in addition no fracture criterion ( $K$-dominant field) is required; crack growth and the crack path are outcomes of the analysis. However, the constraint that cohesive surfaces can only lie along element edges does tend to render crack paths that are biased by the mesh spacing and orientation [24].

Early numerical models for treating discontinuities in finite elements can be traced to the work of Ortiz and co-workers [25] and Belytschko and co-workers [26]. They modelled shear bands as 'weak' (strain) discontinuities that could pass through finite elements using a multifield variational principle. Dvorkin and co-workers [27] considered 'strong' (displacement) discontinuities by modifying the principle of virtual work statement. A unified framework for analysing strong discontinuities by taking into account the softening constitutive law and the interface traction-displacement relation was put forth by Simo and co-workers [28, 29]. Applications and extensions of this approach have been proposed by many researchers todate; for a few related studies, see References [30-33]. In the strong discontinuity approach, the displacement consists of regular and enhanced components, where the enhanced component yields a jump across the discontinuity surface. An assumed enhanced strain variational formulation is used, and the enriched degrees of freedom are statically condensed on an element level to obtain the tangent stiffness matrix for the element. It is to be noted that, in this approach, the discontinuity surface can only terminate on element boundaries. A comprehensive review and comparison of various embedded discontinuity approaches is provided by Jirásek [34].

The use of finite elements to study discrete crack propagation through a polycrystalline microstructure has not been fully explored todate. This stems from the fact that in order to accurately capture the microstructural features and the crack path, refined meshes with continuous adaptive remeshing techniques are required for discrete crack growth simulations. The computational effort and complexity involved is significant even in two-dimensions, and hence the above approach has not attracted wide attention.

A significant improvement in discrete crack modelling has been realized with the development of the extended finite element method (X-FEM) [35-37]. In this approach, the domain is modelled by finite elements with no explicit meshing of the crack surfaces. The location of the crack discontinuity can be arbitrary with respect to the underlying finite element mesh, and quasi-static or fatigue crack propagation simulations can be performed without the need to remesh as the crack advances. In the X-FEM, a discontinuous function (generalized Heaviside step function) and the two-dimensional asymptotic crack-tip displacement fields are added to a standard displacement-based finite element approximation to account for the presence of the crack using the notion of partition of unity [38,39]. A particularly appealing feature is that the finite element framework and its properties (sparsity and symmetry) are retained, and a single-field (displacement) variational principle is used to obtain the discrete equations. The classical finite element degrees of freedom as well as the enriched degrees of freedom are found simultaneously by solving the discrete system. This technique provides an accurate and robust numerical method that removes the need to mesh the crack geometry in both two-dimensional $[35,36]$ and three-dimensional crack modelling [40]. 
In this paper, we present a numerical technique to carry out crack propagation simulations through arbitrary material microstructures. Quasi-static crack propagation is modelled using the X-FEM (Section 2) and microstructures are simulated within the framework of the Potts model for grain growth (Section 3). The microstructural calculations are carried out on a regular lattice, and a novel constrained Delaunay triangulation algorithm is developed to construct the initial finite element mesh of the microstructure. A detailed description of the Delaunay algorithm is presented in Section 4. A standard displacement-based Galerkin method is used to obtain the discrete equations for linear elastostatics (Section 5). The fracture properties of the microstructure are characterized by assuming that the critical fracture energy of the grain boundary $\left(G_{\mathrm{c}}^{\mathrm{gb}}\right)$ is different from that of the grain interior $\left(G_{\mathrm{c}}^{\mathrm{i}}\right)$. The crack growth criterion in the grain interior is governed by the maximum circumferential stress criterion, whereas along the grain boundary, the growth direction is determined by selecting the one that has the maximum value for $G / G_{\mathrm{c}}^{k}$ ( $k$ is either gb or i). In Section 6, numerical simulations of crack propagation through a microstructure are performed for different toughness ratios $G_{\mathrm{c}}^{\mathrm{gb}} / G_{\mathrm{c}}^{\mathrm{i}}$, to study the transition from the intergranular to the transgranular mode of crack growth. The main results and conclusions from this study are discussed in Section 7.

\section{EXTENDED FINITE ELEMENT METHOD}

The X-FEM $[35,36]$ is a numerical method to model internal (or external) boundaries such as holes, inclusions, or multiple cracks, without the need for the mesh to conform to these boundaries. The X-FEM is based on a standard Galerkin procedure, and uses the concept of partition of unity $[38,39]$ to accommodate the internal boundaries in the discrete model. The partition of unity method generalized finite element approximations by presenting a means to embed local solutions of boundary-value problems into the finite element approximation. This idea was exploited in References [41,42] for problems with cracks and holes - the numerical technique was referred to as the generalized finite element method (GFEM).

Partition of unity enrichment for discontinuities and near-tip crack fields was introduced by Belytschko and Black [43]. The displacement enrichment functions for crack problems are functions that span the asymptotic near-tip displacement field. A significant improvement in discrete crack growth modelling without the need for any remeshing strategy was conceived in Reference [35], with further extensions of the technique for modelling holes and branched cracks presented in Reference [36]. The generalized Heaviside step function was proposed as a means to model the crack away from the crack-tip, with simple rules for the introduction of the discontinuous and crack-tip enrichments. This advance has clearly provided an accurate and robust computational tool for modelling discontinuities independent of the mesh geometry in two-dimensions [35,36] and three-dimensions [40]. In addition, recent studies have explored the use of fast marching and level sets for evolving crack discontinuities in three-dimensions within the X-FEM framework: growth of multiple planar cracks are handled using the fast marching method [44,45], whereas non-planar crack growth is carried out using level sets $[46,47]$. The ideas and developments in the X-FEM have had an impact in other related areas. For example, Wells and Sluys [48] proposed a cohesive crack model using finite elements that adopts the notion of partition of unity and the use of the Heaviside step function as an enrichment function to model the displacement discontinuity. 


\subsection{Displacement approximation}

The enrichment of the finite element approximation is described as follows. Consider a point $\mathbf{x}$ of $\mathbb{R}^{d}(d=1-3)$ that lies inside a finite element $e$. Denote the nodal set $\mathbf{N}=\left\{n_{1}, n_{2}, \ldots, n_{m}\right\}$, where $m$ is the number of nodes of element $e .(m=2$ for a linear one-dimensional finite element, $m=3$ for a constant-strain triangle, $m=8$ for a trilinear hexahedral element, etc.) The enriched displacement approximation for a vector-valued function $\mathbf{u}(\mathbf{x}): \mathbb{R}^{d} \rightarrow \mathbb{R}^{d}$ assumes the form:

$$
\mathbf{u}^{h}(\mathbf{x})=\underbrace{\sum_{\substack{I \\ n_{I}}} \phi_{I}(\mathbf{x}) \mathbf{u}_{I}}_{\text {classical }}+\underbrace{\sum_{\substack{J \\ n_{J} \in \mathbf{N}^{g}}} \phi_{J}(\mathbf{x}) \psi(\mathbf{x}) \mathbf{a}_{J}}_{\text {enriched }} \quad\left(\mathbf{u}_{I}, \mathbf{a}_{J} \in \mathbb{R}^{d}\right)
$$

where the nodal set $\mathbf{N}^{g}$ is defined as

$$
\mathbf{N}^{g}=\left\{n_{J}: n_{J} \in \mathbf{N}, \omega_{J} \cap \Omega_{g} \neq \emptyset\right\}
$$

In the above equation, $\omega_{J}=\operatorname{supp}\left(n_{J}\right)$ is the support of the nodal shape function $\phi_{J}(\mathbf{x})$, which consists of the union of all elements with $n_{J}$ as one of its vertices, or in other words the union of elements in which $\phi_{J}(\mathbf{x})$ is non-zero. In addition, $\Omega_{g}$ is the domain associated with a geometric entity such as crack-tip [35], crack surface in three-dimensions [40], or material interface [49]. In general, the choice of the enrichment function $\psi(\mathbf{x})$ that appears in Equation (1) is dependent on the geometric entity.

\subsection{Two-dimensional crack modelling}

The crack is assumed to consist of linear one-dimensional segments. The crack is modelled by enriching the nodes whose nodal shape function support intersects the interior of the crack by a discontinuous function, and enriching the nodes whose nodal shape function supports intersect the crack tip by the two-dimensional asymptotic crack-tip fields. A short description of the selection of nodes for enrichment as well as the computation of enrichment functions follows; for further details, the interested reader can refer to Reference [35]. In addition to the above, partitioning algorithms are also implemented if the crack intersects the finite elements; in this study, the algorithm described in Reference [40] is used with some minor additions for improvement.

2.2.1. Enrichment functions. Consider a single crack in two-dimensions, and let $\Gamma_{\mathrm{c}}$ be the crack surface and $\Lambda_{c}$ the crack tip. The interior of a crack is modelled by the enrichment function $H(\mathbf{x})$, which we refer to as a generalized Heaviside function. The function $H(\mathbf{x})$ takes on the value +1 above the crack and -1 below the crack. More precisely, let $\mathbf{x}^{*}$ be the closest point to $\mathbf{x}$ on the crack $\Gamma_{\mathrm{c}}$, and $\mathbf{n}$ be the normal to the crack segment that contains $\mathbf{x}^{*}$. The $H(\mathbf{x})$ function is then given by

$$
H(\mathbf{x})= \begin{cases}1 & \text { if }\left(\mathbf{x}-\mathbf{x}^{*}\right) \cdot \mathbf{n} \geqslant 0 \\ -1 & \text { otherwise }\end{cases}
$$

To model the crack tip and also to improve the representation of crack-tip fields in fracture computations, crack-tip enrichment functions are used in the element which contains the crack 
tip. The crack-tip enrichment consists of functions which incorporate the radial and angular behaviour of the two-dimensional asymptotic crack-tip displacement field. The use of the crack-tip functions serves two main purposes:

1. If the crack were to terminate in the interior of an element, then enriching the cracktip element with the Heaviside function would be erroneous. This is so, since by such a choice the crack would be modelled as though the segment containing the crack-tip were extended till it intersected the element edge. The crack-tip enrichment functions ensure that the crack terminates precisely at the location of the crack-tip, and hence these functions are clearly required to model the crack for this case.

2. The use of the linear elastic asymptotic crack-tip fields serve as ideal enrichment functions for they possess the correct near-tip behaviour with one of the functions being discontinuous across the crack, and in addition, their use also leads to better accuracy on relatively coarse finite element meshes $[35,40]$.

The crack-tip enrichment functions are:

$$
\boldsymbol{\Phi}(\mathbf{x}) \equiv\left\{\psi_{1}, \psi_{2}, \psi_{3}, \psi_{4}\right\}=\left[\sqrt{r} \cos \frac{\theta}{2}, \sqrt{r} \sin \frac{\theta}{2}, \sqrt{r} \sin \theta \sin \frac{\theta}{2}, \sqrt{r} \sin \theta \cos \frac{\theta}{2}\right]
$$

where $r$ and $\theta$ are polar co-ordinates in the local $x_{\hat{1}}-x_{\hat{2}}$ crack-tip co-ordinate system. Note that the second function in the above equation is discontinuous across the crack.

2.2.2. Selection of enriched nodes. The enriched finite element approximation is given by [35]:

$$
\mathbf{u}^{h}(\mathbf{x})=\sum_{\substack{I \\ n_{I} \in \mathbf{N}}} \phi_{I}(\mathbf{x}) \mathbf{u}_{I}+\sum_{\substack{J \\ n_{J} \in \mathbf{N}^{c}}} \phi_{J}(\mathbf{x}) H(\mathbf{x}) \mathbf{a}_{J}+\sum_{\substack{K \\ n_{K} \in \mathbf{N}^{f}}} \phi_{K}(\mathbf{x})\left(\sum_{l=1}^{4} \psi_{l}(\mathbf{x}) \mathbf{b}_{K}^{l}\right)
$$

The second and third terms on the right-hand side of the above equation are the discontinuity and tip enrichments, respectively. The set $\mathbf{N}^{f}$ consists of those nodes for which the closure of the nodal shape function support intersects the crack tip. The set $\mathbf{N}^{c}$ is the set of nodes whose nodal shape function support is intersected by the crack and which do not belong to $\mathbf{N}^{f}$ :

$$
\begin{aligned}
& \mathbf{N}^{f}=\left\{n_{K}: n_{K} \in \mathbf{N}, \bar{\omega}_{K} \cap \Lambda_{\mathrm{c}} \neq \emptyset\right\}, \\
& \mathbf{N}^{\mathrm{c}}=\left\{n_{J}: n_{J} \in \mathbf{N}, \omega_{J} \cap \Gamma_{\mathrm{c}} \neq \emptyset, n_{J} \notin \mathbf{N}^{f}\right\}
\end{aligned}
$$

\section{POLYCRYSTALLINE MICROSTRUCTURE}

In order to simulate quasi-static crack propagation in a polycrystalline material, a realistic microstructure was first produced using the framework of the Potts model $[12,50]$ for grain growth. Such a procedure was introduced by Srolovitz and co-workers [13-16], and was shown to produce microstructures with grain size and grain topology distributions in 
two- and three-dimensions which were in excellent agreement with experiment. A summary of the grain growth model follows.

A continuum microstructure is mapped onto a regular two-dimensional square lattice containing $N=10,000$ sites. Each lattice site is assigned an integer $s_{i}$, which corresponds to the orientation of the grain in which it is embedded. The number of distinct grain orientations (spins) is $Q$ ( $Q$-state Potts model). Lattice sites which are adjacent to neighbouring sites having different grain orientations are regarded as being adjacent to a grain boundary, whereas a site surrounded by sites with the same grain orientation is in the grain interior. The grain boundary energy is specified by associating a positive energy with grain boundary sites and zero energy for sites in the grain interior, in accordance with the Potts Hamiltonian:

$$
E=J \sum_{i=1}^{N} \sum_{j=1}^{n n(i)}\left(1-\delta_{s_{i} s_{j}}\right)
$$

where $J$ is a constant proportional to the grain boundary energy per unit length, and $\delta_{i j}$ is the Kronecker delta. In the above equation, the summation on $i$ is over all the sites in the lattice, whereas that on $j$ is over the first and second nearest neighbours $n n(i)$ of site $i$. The kinetics of the boundary motion are simulated via a zero-temperature Monte Carlo technique in which a lattice site is selected at random and its orientation is randomly changed to one of the other grain orientations. The change in energy associated with the change in orientation is then evaluated. If the change in energy is less than or equal to zero, the reorientation is accepted; if the energy is raised, the reorientation is rejected.

The microstructures are produced by initially assigning a random value $s_{i}$ of the grain orientation $\left(1 \leqslant s_{i} \leqslant Q\right)$ to each site. Time is directly proportional to Monte Carlo steps: one MCS corresponds to $N$ attempted changes, with the time increment $\Delta t=1 / N$ MCS after every reorientation. The Monte Carlo procedure is executed until the desired grain size is produced. A pseudo-code for the grain growth algorithm is presented in Table I.

\section{DELAUNAY TRIANGULATION OF MICROSTRUCTURE}

The determination of the macroscopic mechanical properties and response of materials from those of their microscopic constituents requires the incorporation of a description of these microstructural features into a continuum-based numerical (finite element) model. The objectoriented finite element analysis program OOF [51,52] was developed to meet this goal. OOF is an image-based program that reads material microstructures in a suitable image format (such as portable pixel map), assigns properties to the microscopic features, and then creates a finite element mesh for further analysis. Alternatively, a microstructure produced from a kinetic Monte Carlo simulation (Potts model) can also be used to generate the finite element mesh [18]. In Reference [18], where OOF is used for the microstructure-meshing, the construction of the finite element mesh is directly based on the bonds between adjacent lattice sites in the Potts model. If neighbouring lattice sites have the same grain orientation (say $A$ ), then properties of the bulk (grain interior) are assigned to the two triangular elements associated with this bond $(A-A)$. On the other hand, if neighbouring lattice sites have different grain orientations (say $A$ and $B$ ), then grain boundary properties (such as crack resistance energy) are assigned to the two triangular elements associated with this bond $(A-B)$. In addition, the properties (such 
Table I. Pseudo-code for Potts grain growth model.

1. read input data - number of lattice sites in each co-ordinate direction $(m)$, total number of orientations (spins) $Q$, and number of MCS steps $t_{\max }$

2. $\quad$ set-up a square lattice of $N=m^{2}$ sites and neighbour list $n n(i)$ for each site $i(i=1 \rightarrow N)$

3. for $i=1 \rightarrow N\{$

- get random number (integer) $s$ between 1 and $Q: s=\operatorname{iran}(1, Q)$;

- assign $s$ as the spin for the site: $\operatorname{spins}[i]=s$;

\}

4. $\quad$ set-up function likes $(i, s)$ that computes the number of neighbours of site $i$ with spin $s$

5. initialize $t=0 ; \Delta t=1 / N$;

6. while $\left(t<t_{\max }\right)\{$

- get a site $i$ at random: $i=\operatorname{iran}(1, N)$;

- spin of site $i$ is: $s_{\text {old }}=\operatorname{spins}[i]$

- choose a new spin at random: $S_{\text {new }}=\operatorname{iran}(1, Q)$;

- compute $\Delta E=\operatorname{likes}\left(i, s_{\text {old }}\right)-\operatorname{likes}\left(i, s_{\text {new }}\right)$;

- if $(\Delta E \leqslant 0)\left\{\right.$ change the spin of site $\left.i: \operatorname{spins}[i]=s_{\text {new }} ;\right\}$

- increment time: $t=t+\Delta t$

\}

7. end

as elastic and thermal) of the element connected to the site with orientation $A$ is assigned the bulk properties of $A$ and likewise for the element connected to the site with orientation $B$. This approach ensures that the whole domain is partitioned into triangles, with grain boundary elements demarcating the transition between two grains of different bulk properties. Clearly, the merits of the method are in its simplicity and ease of implementation. However, we note from the perspective of crack propagation modelling, that the grain boundary which is of zero measure in a two-dimensional continuum description, is replaced by one that has finite width which spans two finite elements. As opposed to relying on finite element (Delaunay) meshes for microstructural analysis, Ghosh and co-workers $[53,54]$ have used the Voronoi tessellation for characterizing and modelling the elasto-plastic response of heterogenous materials with arbitrary size, shape, orientation, and spatial distribution. They use the Voronoi Cell Finite Element Method (VCFEM) [55] for multiscale analysis of heterogeneous materials.

In the work presented in this paper, the crack propagation simulations are performed on a finite element mesh that is constructed using a newly developed constrained Delaunay triangulation algorithm. The input to the meshing algorithm is a polycrystalline microstructure produced by the Potts model, with known spins $s_{i}\left(1 \leqslant s_{i} \leqslant Q, 1 \leqslant i \leqslant N\right)$. A detailed description of the Delaunay meshing algorithm is provided in the following sub-sections.

\subsection{Grain meshing}

There exists a uniquely defined set of grain boundaries for any square lattice with spins assigned to the lattice sites. Let $h$ be the length of an edge $e$ that joins two adjacent lattice sites. If the spin values of these two sites are not equal to each other then a segment of grain boundary must pass through edge $e$. If we assume that this segment should be equidistant from the two sites then it follows that the segment is formed by a straight line connecting the centres of the two lattice cells incident to edge $e$. The union of all such grain boundary 

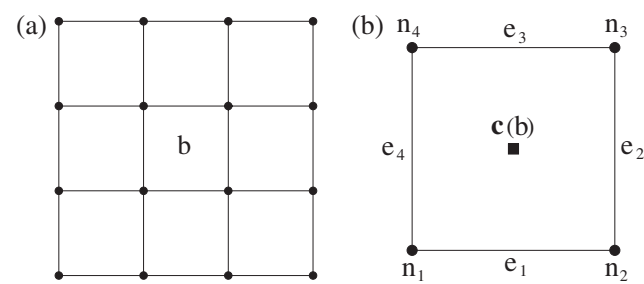

Figure 1. Lattice notations used in the Delaunay meshing algorithm: (a) lattice sites and cell $b$; and (b) sites, edges and centre $\mathbf{c}$ of lattice cell $b$.

segments constitutes the entire set of grain boundaries that separates the microstructure into discrete regions, or grains, each of which is associated with a particular spin value. Each grain boundary consists of a sequence of straight line segments and right angle corners. In other words, the curve defining a grain boundary does not have a continuously turning tangent, and where a tangent is defined, it is either parallel to the $x$ - or $y$-axis.

In order to carry out a finite element computation it is desirable to generate a mesh for each grain so that the elements are of approximately uniform size, and the elements also conform to the shape of the grain boundary curve. Since the grain boundary curve will, in the most general case, have a staircase shape with steps of length $h$ it follows that the linear element size, or element width, will always be $O(h)$. One would like, however, to have the option of specifying an element width that is independent of the lattice edge length $h$.

A more insidious effect of the grain boundary shape lies in the interaction between the grain boundary and a crack path. Since the tangent to the grain boundary can assume one of only two possible directions there is the possibility that the interaction may be incorrectly influenced by the geometry of the problem. There are, therefore, compelling reasons to approximate the true, pixelated grain boundary, by a smooth curve that provides a physically more realistic representation of the grain boundary.

In the next two sub-sections we describe a method to determine the precise shape of each grain boundary curve (i.e. to find the correctly ordered sequence of lattice cell centres through which the true grain boundary passes). Next, we present details of the curve smoothing technique and in the following sub-section we provide a brief discussion of the meshing algorithm.

\subsection{Data structures}

Let the lattice have $N$ sites and let $\mathbf{r}(n)$ be the position vector associated with the $n$th site. Each site is assigned an integer valued number known as the spin. Write $s_{n}$ for the spin of the $n$th site. Let $b$ denote a lattice cell whose sites have the addresses $n_{1}, n_{2}, n_{3}, n_{4}$ and define the set $V(b)=\left\{n_{1}, n_{2}, n_{3}, n_{4}\right\}$. Let $\mathbf{c}(b)$ be the position vector whose co-ordinates are the $x$ - and $y$-components of the centre of the lattice cell $b$. In Figure 1(a), a schematic of a $4 \times 4$ lattice is shown with the above lattice notations indicated in Figure 1(b) for a particular lattice cell $b$. Thus,

$$
\mathbf{c}(b)=\frac{1}{4}\left(\mathbf{r}\left(n_{1}\right)+\mathbf{r}\left(n_{2}\right)+\mathbf{r}\left(n_{3}\right)+\mathbf{r}\left(n_{4}\right)\right)
$$


Now create a butterfly data structure edge $(i, e), i=1,2,3,4$, such that for each lattice edge $e$, edge $(1, e)=n_{1}$, edge $(2, e)=n_{2}$, where $n_{1}$ and $n_{2}$ are the lattice site addresses associated with the end-points of $e$. Set edge $(3, e)=b_{1}$ and edge $(4, e)=b_{2}$, where $b_{1}$ and $b_{2}$ are the two lattice cells that are incident to $e$ (Figure 1(b)). If there is only one lattice cell $b$ incident to $e$, we call this a boundary edge and set edge $(3, e)=b$ and edge $(4, e)=0$. We also associate with each lattice cell $b$ the set $S(b)=\left\{e_{1}, e_{2}, e_{3}, e_{4}\right\}$, where the $e_{i}(i=1,2,3,4)$ are the edge addresses of the four sides of the lattice cell $b$.

Given an edge $e$, let $n_{1}=\operatorname{edge}(1, e)$ and $n_{2}=\operatorname{edge}(2, e)$, and define the function

$$
g(e)=1-\delta_{s_{n_{1}} s_{n_{2}}}
$$

where $\delta_{i j}$ is the Kronecker delta. In other words, $g(e)$ takes the value 1 if a segment of a grain boundary passes through edge $e$. This segment connects the centres $\mathbf{c}\left(b_{1}\right)$ and $\mathbf{c}\left(b_{2}\right)$ of the two lattice cells $b_{1}$ and $b_{2}$ that are incident to this edge. If $e$ is a boundary edge for which $g(e)=1$ then the associated grain boundary segment starts at the mid-point of the edge and ends at the point $\mathbf{c}(b)$ where $b=\operatorname{edge}(3, e)$.

We can now classify each lattice cell $b$ as either:

1. Interior if no grain boundary passes through $b$ (i.e. if $g(e)=0 \forall e \in S(b)$ );

2. boundary if a single grain boundary passes through $b$ (i.e. if $g(e)=1$ for two edges in $S(b)$ and $g(e)=0$ for the remaining two edges in $S(b))$;

3. a junction if one or more boundaries start and/or terminate in $b$. In this case $g(e)=1$ for 3 or 4 edges in $S(b)$.

For each lattice cell $b$ we assign an integer-valued function $f(b)$ that counts the number of edges $e \in S(b)$ for which $g(e)=1$. Thus,

$$
f(b)= \begin{cases}0 & \text { if } b \text { is in the interior } \\ 2 & \text { if } b \text { is a boundary } \\ 3 \text { or } 4 & \text { if } b \text { is a junction }\end{cases}
$$

The case $f(b)=4$ is mentioned so as to cover all the possibilities. We do emphasize that the case $f(b)=4$ does not arise in microstructural evolution processes such as grain growth in isotropic systems, since it is a thermodynamically (energetically) unfavourable configuration and splits into two triple junctions [56]. The case $f(b)=3$ (triple junction) is energetically favourable. In addition, we note that $f(b)$ cannot assume the value 1 since a grain boundary cannot start or terminate inside a lattice cell unless the terminal point is incident to at least one other grain boundary that enters $b$.

This leads to the following classification of the grain boundary curves into two distinct types:

1. The curve starts at a boundary edge. This curve may end either (a) inside a lattice cell (i.e. at a junction), or (b) at another boundary edge.

2. The curve starts and ends at a junction.

There exists a third possibility, namely, the grain boundary curve is isolated (i.e. $f(b)=2$ for each lattice cell $b$ through which the curve passes). However, this case is not physical since grain boundaries may only end on other grain boundaries; hence this type is not taken into consideration. 


\subsection{Assembling grain boundary segments}

The individual segments of each grain boundary can be assembled in the correct order by using the butterfly edge data structure to traverse the lattice. It is convenient to maintain a list of all edges for which $g(e)=1$ and which have not yet been examined. Thus, we start by establishing the set $G=\{e: g(e)=1\}$ and remove an edge $e$ from $G$ after this edge has been examined. The task is finished and the complete set of grain boundary curves has been found when the cardinality of $G$ is zero.

We first assemble all type 1 grain boundaries; these start on the lattice boundary. Then, assuming $G$ is not yet empty, we assemble the remaining grain boundaries; these must necessarily be type 2 .

Consider, for example, the assembly of a type 2 grain boundary curve $\Gamma$. Let $b_{0}$ be the junction at which the $\Gamma$ starts. Search through the edges $e \in S\left(b_{0}\right)$ to find a side $e^{\prime} \in S\left(b_{0}\right) \cap G$. Write $b_{1}$ for the lattice cell adjacent to $b_{0}$ that is also incident to $e^{\prime}$. If $b_{1}$ is a junction then $b_{1}$ is the final lattice cell associated with $\Gamma$. If $b_{1}$ is not a junction then the procedure is repeated. Let $b_{m}, m \geqslant 1$ be the final lattice cell (i.e. the first junction found by this search procedure). Then the sequence of lattice cells $\left\{b_{k}: k=0, \ldots, m\right\}$ defines $\Gamma$, and the segment of $\Gamma$ that connects lattice cell $k$ to lattice cell $k+1$ is given by

$$
\mathbf{r}(t)=\mathbf{c}\left(b_{k}\right)+\left[\mathbf{c}\left(b_{k+1}\right)-\mathbf{c}\left(b_{k}\right)\right](t-k h), \quad k h \leqslant t \leqslant(k+1) h
$$

where $h$ is the lattice edge length. Apart from minor adjustments, type 1 grain boundary curves are also handled in essentially the same way.

\subsection{Smoothing of grain boundaries}

Each grain boundary curve $\Gamma$ is approximated by cubic polynomials $x(t)$ and $y(t)$ that pass through the two end-points of $\Gamma$. Each of these cubics has two remaining degrees of freedom which we can adjust to achieve a least-squares fit. Let $b_{0}$ be the lattice cell at the start of $\Gamma$ and $b_{m}$ be the lattice cell at the other end-point of $\Gamma$. Write $t_{k}=k h, k=0, \ldots, m$ and let $\left(x_{k}, y_{k}\right)$ be the $x$ and $y$ components of $\mathbf{c}\left(b_{k}\right)$, the centre of lattice cell $b_{k}$. We seek a cubic polynomial that passes through $\left(x_{0}, y_{0}\right)$ and $\left(x_{m}, y_{m}\right)$ and is the best fit to the remaining points $\left(x_{k}, y_{k}\right), k=1, \ldots, m-1$ in the least-squares sense. Define

$$
\tau=\frac{t-t_{0}}{t_{m}-t_{0}} \quad \text { and } \quad \tau_{k}=\frac{t_{k}-t_{0}}{t_{m}-t_{0}}, \quad k=0, \ldots, m
$$

Then the $x$ and $y$ components of the smoothed curve are given by

$$
\begin{aligned}
& x(\tau)=a\left(\tau^{3}-\tau\right)+b\left(\tau^{2}-\tau\right)+\left(x_{m}-x_{0}\right) \tau+x_{0} \\
& y(\tau)=c\left(\tau^{3}-\tau\right)+d\left(\tau^{2}-\tau\right)+\left(y_{m}-y_{0}\right) \tau+y_{0}
\end{aligned}
$$

The mean square error is

$$
E(a, b ; c, d)=\sum_{k=1}^{m-1}\left[\left(x\left(\tau_{k}\right)-x_{k}\right)^{2}+\left(y\left(\tau_{k}\right)-y_{k}\right)^{2}\right]
$$


Setting

$$
\frac{\partial E}{\partial a}=0, \quad \frac{\partial E}{\partial b}=0, \quad \frac{\partial E}{\partial c}=0, \quad \frac{\partial E}{\partial d}=0
$$

we find the coefficients $a, b, c$, and $d$ by solving the following two sets of equations:

$$
\left(\begin{array}{ll}
\alpha & \beta \\
\beta & \gamma
\end{array}\right)\left(\begin{array}{l}
a \\
b
\end{array}\right)=\left(\begin{array}{l}
p \\
q
\end{array}\right), \quad\left(\begin{array}{ll}
\alpha & \beta \\
\beta & \gamma
\end{array}\right)\left(\begin{array}{l}
c \\
d
\end{array}\right)=\left(\begin{array}{l}
r \\
s
\end{array}\right)
$$

where

$$
\alpha=\sum_{k=1}^{m-1}\left(\tau_{k}^{2}-1\right)^{2} \tau_{k}^{2}, \quad \beta=\sum_{k=1}^{m-1}\left(\tau_{k}^{2}-1\right)\left(\tau_{k}-1\right) \tau_{k}^{2}, \quad \gamma=\sum_{k=1}^{m-1}\left(\tau_{k}-1\right)^{2} \tau_{k}^{2}
$$

and

$$
\begin{aligned}
& p=\sum_{k=1}^{m-1}\left[x_{k}-x_{0}-\left(x_{m}-x_{0}\right) \tau_{k}\right]\left(\tau_{k}^{2}-1\right) \tau_{k} \\
& q=\sum_{k=1}^{m-1}\left[x_{k}-x_{0}-\left(x_{m}-x_{0}\right) \tau_{k}\right]\left(\tau_{k}-1\right) \tau_{k}
\end{aligned}
$$

The terms $r$ and $s$ are determined in a similar way on replacing $x_{k}$ by $y_{k}, x_{0}$ by $y_{0}$, and $x_{m}$ by $y_{m}$ in the definition of $p$ and $q$.

\subsection{Mesh generation}

The domain to be meshed consists of the interior of the lattice boundary. It is important, however, that the smoothed grain boundaries are maintained within the final mesh. The lattice boundary and grain boundaries are discretized and approximated by a series of contiguous straight line segments whose length is chosen by the user. These straight line segments form the set of mesh boundary edges, and their end-points the set of mesh boundary points. A constrained Delaunay triangulation of this collection of edges and points is then created. It is known that the constrained Delaunay triangulation of any planar domain, containing a given set of prescribed edges, always exists [57]. This initial triangulation is generated by the Tanemura-Ogawa-Ogita algorithm [58].

The initial mesh is then refined to create a mesh of good quality triangles that also conforms to all boundary edges. The refinement is based on incremental point insertion using the Bowyer-Watson algorithm [59,60]. The quality of the final mesh will depend on the placement of the interior mesh points. Several possibilities have been considered for selecting the position of point placement (e.g. at element barycentres [61], along edges [62], at element circum-centres [63] or along Voronoi segments [64]). The circum-centre point insertion method generates provably good quality meshes in two-dimensions $[65,66]$. The Voronoi segment method $[64,66]$ works extremely well for planar domains and has been shown to generate meshes whose triangles are mostly close to equilateral in shape [66]. 
The decision, whether or not to refine a particular region of the mesh, is based on a comparison between the actual local length scale $\ell$ (e.g. element width, circum-radius) and the desired length scale specified by a scalar variable $\rho$ called the length density function. The value of the mesh density function at each point on the boundary is computed as the average length of the incident boundary edges and values of $\rho$ at interior points are found by linear interpolation over the triangles. In the computations carried out in this paper, a user-defined constant value of $\rho$ is specified for all points on the boundary. Hence, the value of $\rho$ for all interior points in the domain is also approximately the same constant. If the value of $\rho$ at any position in the mesh is less than the actual local length scale $\ell$ then the mesh is refined by the insertion of an extra point followed by a local mesh reconstruction using the incremental Delaunay algorithm. It can be shown that the maximum circum-radius of the Delaunay triangulation is monotone decreasing if each new mesh point is always placed in a triangle whose circum-radius has the maximum value [66]. It follows that the circum-radii of all triangles in the mesh will eventually fall below the value determined by the desired local length scale $\rho$ and the algorithm is therefore guaranteed to halt after a finite number of steps.

In Figure 2, examples of the finite element meshes generated by the microstructure-meshing algorithm are presented. The initial triangulation of the domain which preserves all grain boundary edges is shown in Figure 2(a) and (b) for $\rho=0.1$ and 0.04 , respectively. After carrying out the refinement procedure for a given $\rho$, the final triangulation of the microstructure is shown in Figure 2(c) $(\rho=0.1)$ and Figure 2(d) $(\rho=0.04)$. The mesh in Figure 2(c) consists of 395 three-noded constant strain triangular elements, whereas the mesh presented in Figure 2(d) has 2002 elements.

\section{GOVERNING EQUATIONS AND WEAK FORM}

Consider a body $\Omega \subset \mathbb{R}^{2}$, with boundary $\Gamma$. The boundary $\Gamma$ consists of the sets $\Gamma_{u}$ (essential boundary), $\Gamma_{t}$ (natural boundary), and $\Gamma_{\mathrm{c}}$ (internal crack), such that $\Gamma=\Gamma_{u} \cup \Gamma_{t} \cup \Gamma_{\mathrm{c}}$. The crack $\Gamma_{c}$ is assumed to be traction-free.

The field equations of elastostatics are:

$$
\begin{aligned}
\boldsymbol{\nabla} \cdot \boldsymbol{\sigma}+\mathbf{b} & =0 \quad \text { in } \Omega \\
\boldsymbol{\sigma} & =\mathbf{C}: \boldsymbol{\varepsilon} \\
\boldsymbol{\varepsilon} & =\boldsymbol{\nabla}_{\mathrm{s}} \mathbf{u}
\end{aligned}
$$

where $\nabla_{\mathrm{s}}$ the symmetric gradient operator, $\mathbf{u}$ the displacement vector, $\boldsymbol{\varepsilon}$ the small strain tensor, $\boldsymbol{\sigma}$ the Cauchy stress tensor, $\mathbf{b}$ the body force vector per unit volume, and $\mathbf{C}$ the tensor of elastic moduli for a homogeneous isotropic material.

The essential and natural boundary conditions are:

$$
\begin{aligned}
& \mathbf{u}=\overline{\mathbf{u}} \quad \text { on } \Gamma_{u} \\
& \boldsymbol{\sigma} \cdot \mathbf{n}=\overline{\mathbf{t}} \text { on } \Gamma_{t} \\
& \boldsymbol{\sigma} \cdot \mathbf{n}=0 \quad \text { on } \Gamma_{\mathrm{c}}
\end{aligned}
$$



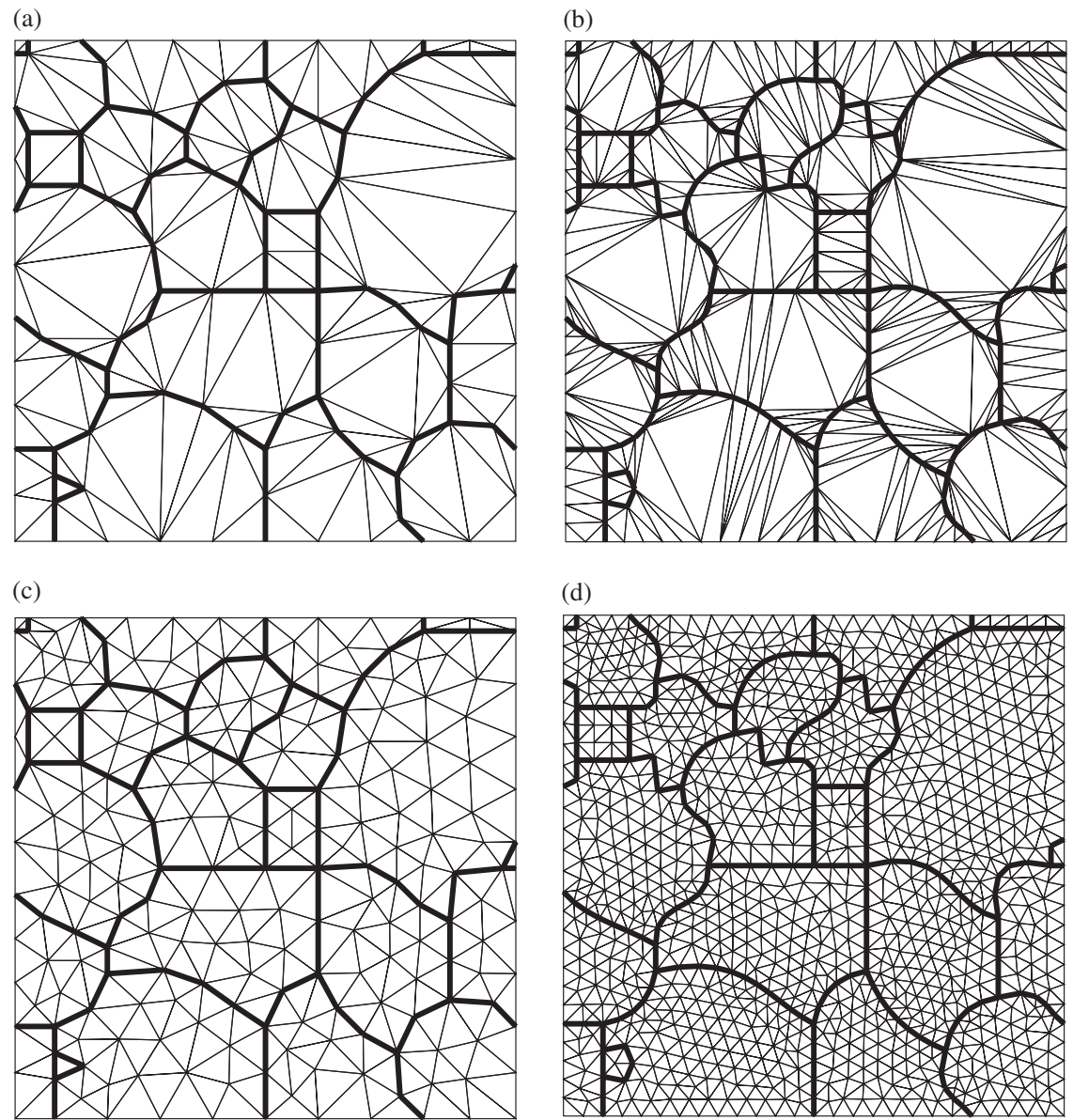

Figure 2. Delaunay triangulation of polycrystalline microstructure: (a) initial boundary conforming triangulation $(\rho=0.1)$; (b) initial boundary conforming triangulation $(\rho=0.04)$; (c) final triangulation $(\rho=0.1)$; and (d) final triangulation $(\rho=0.04)$.

where $\mathbf{n}$ is the unit outward normal to $\Omega$, and $\overline{\mathbf{u}}$ and $\overline{\mathbf{t}}$ are prescribed displacements and tractions, respectively. Note that Equation $(19 \mathrm{c})$ imposes the condition that the crack $\Gamma_{\mathrm{c}}$ be traction-free [43].

The weak form (principle of virtual work) for linear elastostatics is stated as: Find $\mathbf{u}^{h} \in \mathbf{V}^{h}$ such that

$$
\int_{\Omega^{h}} \boldsymbol{\sigma}\left(\mathbf{u}^{h}\right): \boldsymbol{\varepsilon}\left(\mathbf{v}^{h}\right) \mathrm{d} \Omega=\int_{\Omega^{h}} \mathbf{b} \cdot \mathbf{v}^{h} \mathrm{~d} \Omega+\int_{\Gamma_{t}^{h}} \overline{\mathbf{t}} \cdot \mathbf{v}^{h} \mathrm{~d} \Gamma \quad \forall \mathbf{v}^{h} \in \mathbf{V}_{0}^{h}
$$

where $\mathbf{u}^{h}(\mathbf{x}) \in \mathbf{V}^{h}$ and $\mathbf{v}^{h}(\mathbf{x}) \in \mathbf{V}_{0}^{h}$ are the approximating trial and test functions used in the $\mathrm{X}$-FEM. The space $\mathbf{V}^{h}$ is the enriched finite element space that satisfy the essential boundary conditions, and which include basis functions that are discontinuous across the crack surfaces. 


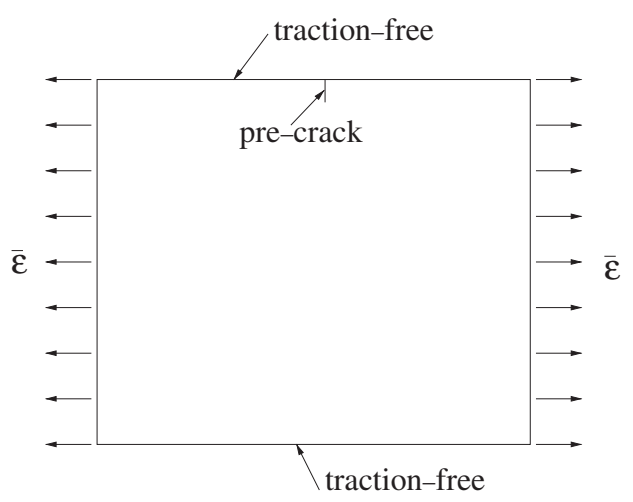

Figure 3. Model geometry and boundary conditions.

The space $\mathbf{V}_{0}^{h}$ is the corresponding space with homogeneous essential boundary conditions. The trial and test functions, which are based on Equation (5) are used in a standard Galerkin procedure to obtain the discrete equation $[35,36]$.

\section{NUMERICAL EXAMPLES}

We first describe the simulation procedure for crack growth through a microstructure. Next, we present the numerical simulation results for crack propagation in a polycrystalline material for different grain boundary toughness, showing the transition from the intergranular to the transgranular mode of fracture. A detailed study that takes into account the statistical variations in the microstructure, and the dependence of grain size and toughness on crack propagation is currently under investigation; in this paper, we focus on the development and implementation of the numerical algorithms together with a few crack propagation simulations that demonstrate the promise and potential of the proposed approach.

\subsection{Simulation procedure}

A polycrystalline microstructure is obtained using the Potts grain growth model outlined in Section 3. The polycrystal is assumed to be elastically homogeneous - all grains and grain boundaries have the same elastic constants $(E$ and $v)$. A finite element mesh of the microstructure is generated using the Delaunay algorithm described in Section 4. The problem domain is a square of edge length $L$. An initial pre-crack of size $0.02 L$ is introduced along a grain boundary that emanates from $x_{1}=0.5 \mathrm{~L}$ on the top surface. The top and bottom surfaces are traction-free; uniaxial strain is applied in the $x_{1}$-direction by fixing the left edge and imposing displacement boundary conditions on the right edge (Figure 3). A user-defined crack growth increment $\Delta a_{\max }$ is also prescribed as an input. At any strain step $j$, the X-FEM solution is obtained for an initial (guess) applied strain that is taken from the previous converged step $\bar{\varepsilon}_{j}^{g}=\bar{\varepsilon}_{j-1}\left(\bar{\varepsilon}_{0}=1\right)$. Fracture parameters such as the mode I and mode II stress intensity factors are computed using the domain form [67,68] of the interaction integral [69]; see Reference [35] for details on the stress intensity factor computations in the X-FEM. The strain energy 
release rate $G$ under plane strain conditions is related to the stress intensity factors through Irwin's relation:

$$
G=\frac{\left(1-v^{2}\right)\left(K_{\mathrm{I}}^{2}+K_{\mathrm{II}}^{2}\right)}{E}
$$

where $E$ and $v$ are Young's modulus and Poisson's ratio, respectively, of the material. Depending on the crack-tip position, one of two cases may occur, namely

(A) Crack-tip is in the grain interior.

(B) Crack-tip is on a grain boundary.

If the crack-tip is in the grain interior, then the following steps are carried out:

(A.1) The maximum circumferential stress criterion [70] is used to determine the crack growth direction $\left(\theta_{g}=\theta_{h}\right)$ :

$$
\theta_{h}=2 \tan ^{-1} \frac{1}{4}\left(\frac{K_{\mathrm{I}}}{K_{\mathrm{II}}} \pm \sqrt{\left(\frac{K_{\mathrm{I}}}{K_{\mathrm{II}}}\right)^{2}+8}\right) \quad \text { if } K_{\mathrm{II}} \neq 0
$$

where $\theta_{h}$ is the crack growth angle in the local crack-tip co-ordinate system.

(A.2) The crack increment in this case is given by

$$
\Delta a=\min \left(\Delta a_{\max }, \Delta a_{\mathrm{gb}}, \Delta a_{\mathrm{hull}}\right)
$$

where $\Delta a_{\mathrm{gb}}$ is the distance from the crack-tip to the nearest grain boundary along $\theta_{h}$ and $\Delta a_{\text {hull }}$ is the distance from the crack-tip to the convex hull along $\theta_{h}$.

If the crack-tip is on a grain boundary, then the steps involved are:

(B.1) All directions along the grain boundary from the crack-tip are considered along with the maximum circumferential stress direction $\theta_{\mathrm{h}}$.

The maximum allowable crack increment $\Delta a_{m}$ is determined from the following equation:

$$
\Delta a_{m}=\min \left(\Delta a_{\mathrm{max}}, \Delta a_{\mathrm{gb}}, \Delta a_{\mathrm{hull}}, \Delta a_{\mathrm{gbj}}\right)
$$

where $\Delta a_{\mathrm{gbj}}$ is the distance to the nearest grain boundary junction along $\theta_{\mathrm{gb}}$. The crack is advanced by $10 \%$ of $\Delta a_{m}$ in each permissible direction and the strain energy release rate $G$ is computed for each case. We disregard any of the grain boundary directions that lead to crack closure. The crack faces are not in contact if

$$
\left(\mathbf{u}^{+}-\mathbf{u}^{-}\right) \cdot \mathbf{n}^{+}>0
$$

in the middle of the crack segment which contains the crack-tip. In the above equation, $\mathbf{u}^{+}$and $\mathbf{u}^{-}$are the displacement vector solutions above and below the crack segment, respectively, and $\mathbf{n}^{+}$is the unit normal to the crack segment. 
(B.3) The ratios $G / G_{\mathrm{c}}^{k}$ are computed, where $k=\mathrm{i}$ (grain interior) or $k=\mathrm{gb}$ (grain boundary), and the one with the largest ratio is chosen as the growth direction.

(B.4) If a grain boundary direction is selected as the growth direction $\left(\theta_{g}=\theta_{\mathrm{gb}}\right)$, then the crack increment in this case is given by

$$
\Delta a=\min \left(\Delta a_{\mathrm{max}}, \Delta a_{\mathrm{gbj}}\right)
$$

Once the growth direction and crack growth increment are determined in step $j$, the critical applied strain for step $j$ is computed so that crack growth takes place when $G \geqslant G_{\mathrm{c}}^{k}(k=\mathrm{i}$ or $k=\mathrm{gb})$. Since the problem is linear elastic, the strain energy release rate is proportional to the square of the applied strain. Hence if $G<G_{\mathrm{c}}^{k}(k=\mathrm{i}$ or $k=\mathrm{gb})$ at step $j(j>1$, since for $j=1$ the critical applied strain is computed even if $G>G_{\mathrm{c}}^{k}$ ), the critical applied strain $\bar{\varepsilon}_{j}$ is computed using:

$$
\bar{\varepsilon}_{j}=\sqrt{\frac{G_{\mathrm{c}}^{k}}{G}} \bar{\varepsilon}_{j}^{g}
$$

After the X-FEM analysis has been carried out with the critical applied strain for the appropriate growth direction and crack growth increment, the simulation proceeds to the next $(j+1)$ step and the above sequence is repeated. The simulation is carried out till fracture of the specimen. In all simulations, plane strain conditions are assumed, with Young's modulus $E=10^{5}$ and Poisson's ratio $v=0.3$ used in the computations.

\subsection{Numerical simulations}

In the numerical simulations, we study the dominant mode of crack propagation in terms of the fracture toughness (critical fracture energy) of the grain boundary vis-à-vis that of the grain interior. Let $R=G_{\mathrm{c}}^{\mathrm{gb}} / G_{\mathrm{c}}^{\mathrm{i}}$ denote the ratio of the critical fracture energy of the grain boundary to that of the grain interior. In all simulations, a unit square lattice was used in the Potts grain growth model with $N=10^{4}$ (lattice sites) and $Q=100$ (spins). The grain sizes in the polycrystalline microstructures were obtained after executing the kinetic Monte Carlo simulation for $10^{4}$ MCS steps. The scalar length density function $\rho=0.02$ was used to construct the finite element mesh from the microstructure. The crack propagation simulations were performed using the procedure outlined in Section 6.1.

In Figure 4, the grain boundaries and the crack path are shown for the case of very weak grain boundaries $(R=0.1)$. As one might expect, the fracture mode is entirely intergranular, with the crack propagating entirely along the grain boundaries. Since the extreme values of $R$ will result in intergranular $(0<R<0.2)$ or transgranular $(0.8<R<1)$ fracture paths, we focus on intermediate values of $R$ for the remaining simulations.

We consider three different values of $R$ in our crack propagation studies: $R=0.3,0.5,0.7$. In addition, for each case the percentage of the crack length that lies along grain boundaries (i.e. the intergranular fraction, IG) is also indicated in the plots. We show results obtained for two different material microstructures to emphasize the inherent statistical variability of these type of microstructures. Figures 5 and 6 show results obtained for two different microstructures. Clearly, the same trends are evident in both cases - the transition from an intergranular to a transgranular mode of fracture is observed as $R$ is increased. For $R=0.3$, both Figures 5 (a) and 6(a) illustrate a predominantly intergranular mode of fracture; the growth is mixed mode 


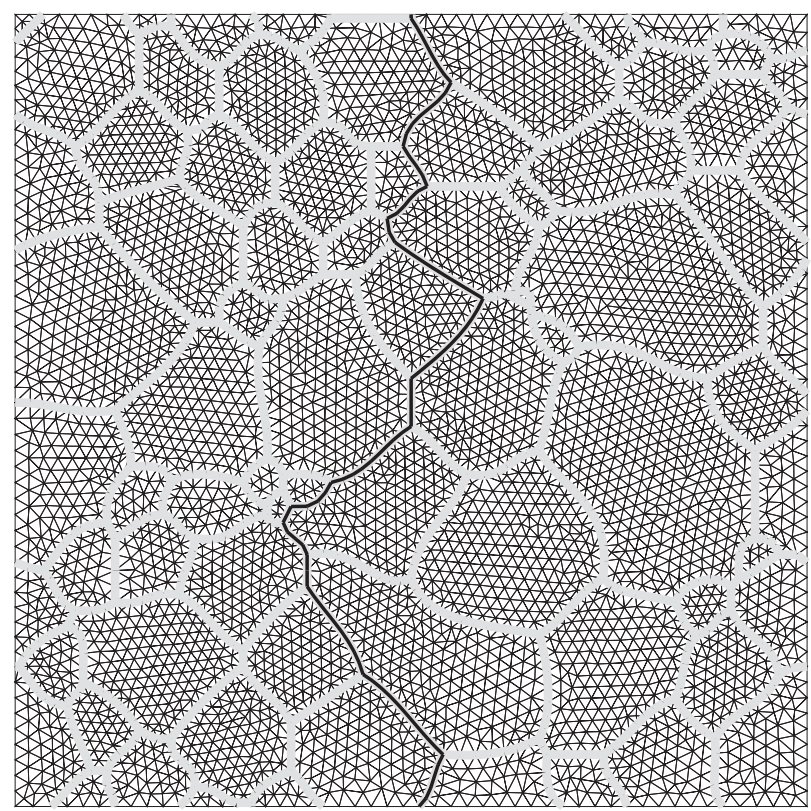

Figure 4. Intergranular fracture $(R=0.1)$.

(intergranular and transgranular) when $R=0.5$, as shown in Figures $5(\mathrm{~b})$ and $6(\mathrm{~b})$; and the crack path is transgranular dominant (cleavage) in Figures $5(\mathrm{c})$ and $6(\mathrm{c})$ for $R=0.7$. To give a better pictorial comparison of the crack paths, all cases are shown for each microstructure in Figures 5(d) and 6(d).

\section{CONCLUSIONS}

We presented a numerical model for crack propagation through a realistic polycrystalline material microstructure. The X-FEM [35] was adopted as the numerical technique of choice. In the X-FEM, a discontinuous function and the two-dimensional asymptotic crack-tip displacement fields are added to the finite element approximation to account for the crack using the notion of partition of unity. This enables the domain to be modelled by finite elements with no explicit meshing of the crack surfaces. Hence, quasi-static crack propagation can be simulated without any user-intervention or the need to remesh as the crack advances.

The methodology used to carry out crack propagation simulations through a polycrystalline microstructure consisted of three main ingredients:

1. Unlike in many existing simulations of brittle fracture, a continuum description of a polycrystal was employed. This was based on a realistic microstructure produced using the Potts grain growth model.

2. A novel constrained Delaunay algorithm was developed to construct a finite element mesh of the material microstructure. The meshing algorithm accommodated grain boundary smoothing using a least-squares cubic polynomial fit through adjacent grain boundary 
(a)

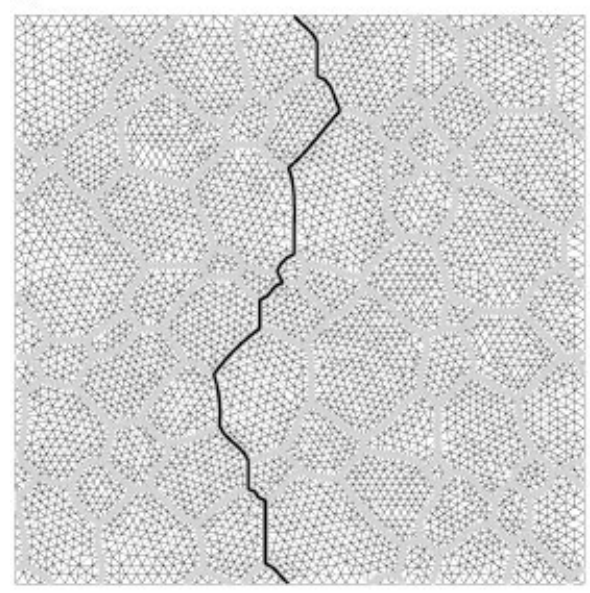

(c)

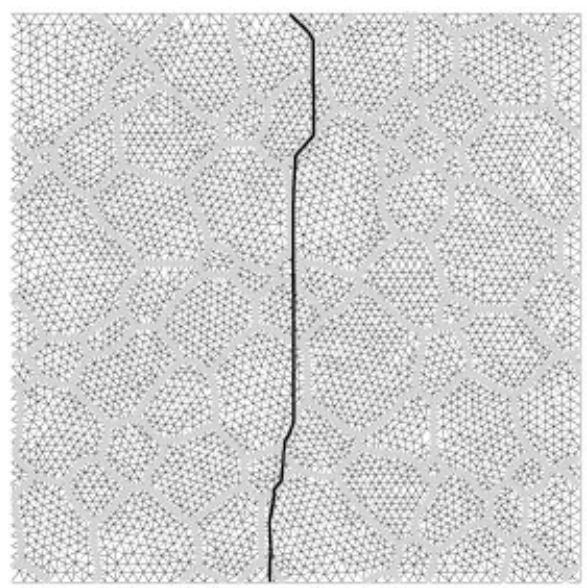

(b)

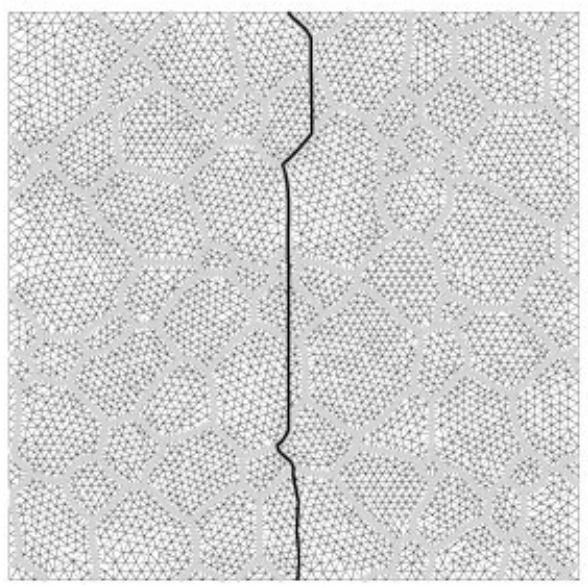

(d)

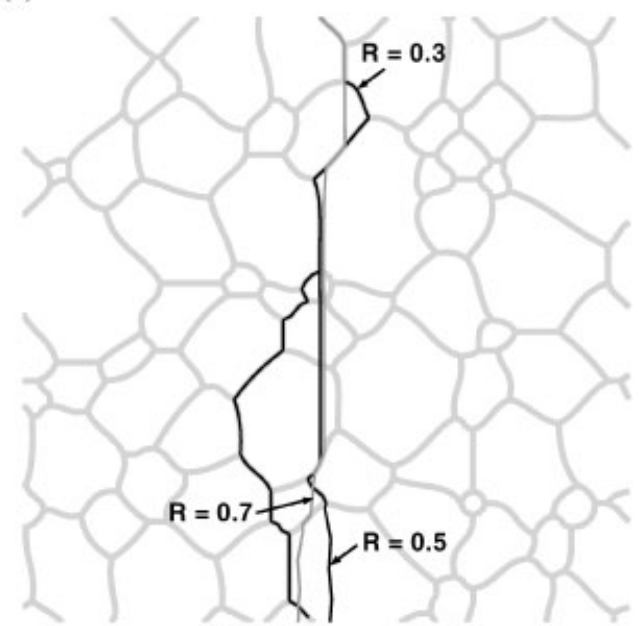

Figure 5. Crack propagation through polycrystalline microstructure I. The thin grey lines delineate the finite element mesh, the artificially thickened (for clarity) grey lines represent the grain boundaries and the black line indicates the crack path: (a) $R=0.3(\mathrm{IG}=100 \%) ;(\mathrm{b}) R=0.5$ ( $\mathrm{IG}=42 \%)$; (c) $R=0.7(\mathrm{IG}=21 \%)$; and (d) all cases.

vertices; in addition, the flexibility of a user-specified scalar local mesh density $\rho$ was also provided.

3. A quasi-static crack growth criteria was proposed that was based on the local mechanical state at the crack-tip, which was driven by the direction of maximum circumferential stress in the grain interior and along the local direction with maximum energy release rate from a grain boundary: the toughness (critical energy) of the grain boundary and the grain interior were assumed to be different. Crack growth took place if the condition $G \geqslant G_{\mathrm{c}}$ was met, where $G_{\mathrm{c}}$ depended on the microstructural constituents. 
(a)

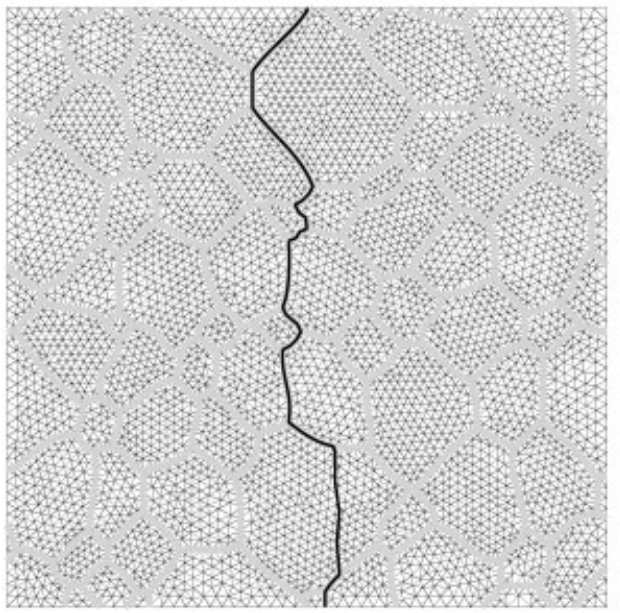

(c)

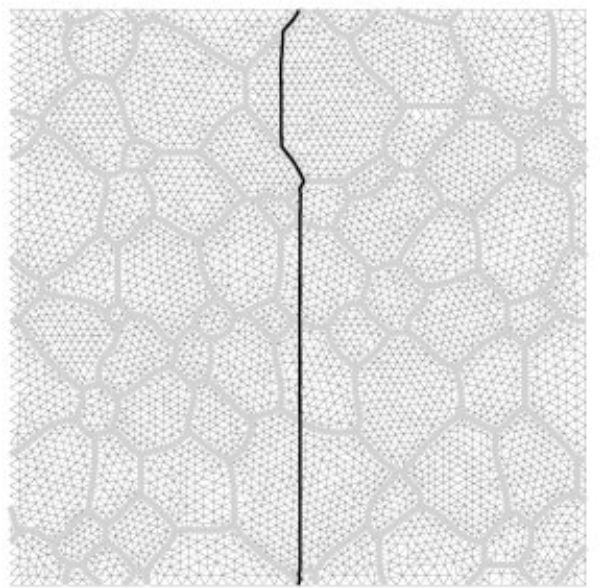

(b)

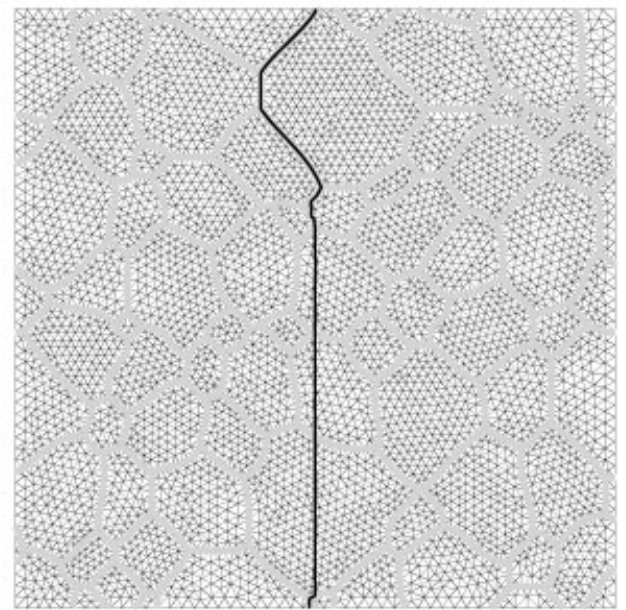

(d)

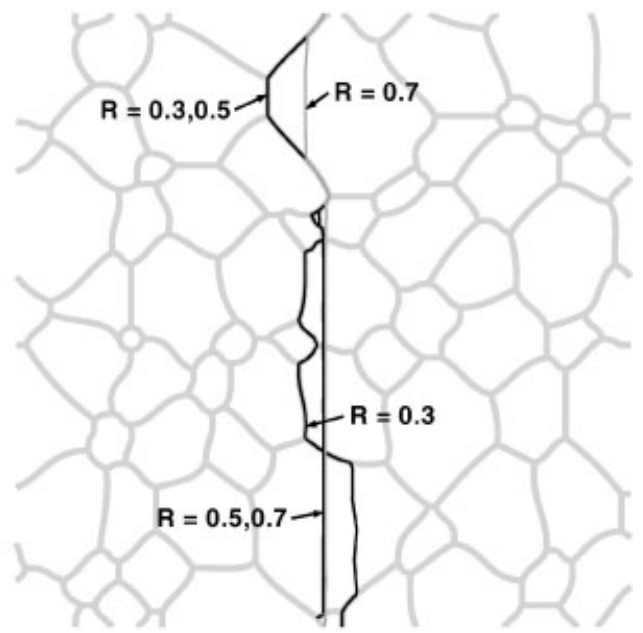

Figure 6. Crack propagation through polycrystalline microstructure II: (a) $R=0.3$ (IG $=81 \%$ ); (b) $R=0.3(\mathrm{IG}=40 \%)$; (c) $R=0.7(\mathrm{IG}=13 \%)$; and (d) all cases.

Crack propagation simulations were presented as a function of the ratio of the critical fracture energy of the grain boundary to that of the grain interior: $R=G_{\mathrm{c}}^{\mathrm{gb}} / G_{\mathrm{c}}^{\mathrm{i}}$. In the simulations, we observed the transition from an intergranular to a transgranular mode of fracture as the grain boundary toughness was increased, which is in qualitative agreement with the results obtained in previous studies $[11,19]$. This study has demonstrated the utility and potential of the XFEM as a computational fracture tool to study complex failure mechanisms in polycrystalline materials. 


\section{ACKNOWLEDGEMENTS}

The financial support of Idaho National Engineering Laboratory (Contract No. KOO-182412) to Princeton University, and the partial support to J.P. and T.J.B. from the National Science Foundation through contract NSF-9988788, Dr Jorn Larsen-Basse Program Manager, is gratefully acknowledged. The authors also thank Mark Miodownik and Elizabeth Holm for providing the Potts model grain growth code.

\section{REFERENCES}

1. Watanabe T. The impact of grain boundary character distribution on fracture in polycrystals. Materials Science and Engineering A 1994; 176:39-49.

2. Aust KT, Erb U, Palumbo G. Interface control for resistance to intergrangular cracking. Materials Science and Engineering A 1994; 176:329-334.

3. Kumar M, King WE, Schwartz AJ. Modifications to the microstructural topology in f.c.c. materials through thermomechanical processing. Acta Materialia 2000; 48(9):2081-2091.

4. Beale PD, Srolovitz DJ. Elastic fracture in random materials. Physics Review B 1998; 37(10):5500-5507.

5. Curtin WA, Scher H. Brittle fracture of disordered materials. Journal of Materials Research 1990; 5(3): $535-553$.

6. Curtin WA, Scher H. Mechanics modeling using a spring network. Journal of Materials Research 1990; 5(3):554-562.

7. Jagota A, Bennison SJ. Spring-network and finite-element models for elasticity and fracture. In Nonlinearity and Breakdown in Soft Condensed Matter, Springer Lecture Notes in Physics, Bardhan KK, Chakrabarti BK, Hansen A (eds), vol. 437. Springer: Berlin, 1994, pp. 186-201.

8. Jagota A, Bennison SJ. Element breaking rules in computational models for brittle fracture. Modelling and Simulation in Materials Science and Engineering 1995; 3(4):485-501.

9. Schlangen E, Garboczi EJ. New method for simulating fracture using an elastically uniform random geometry. International Journal of Engineering Science 1996; 34(10):1131-1144.

10. Bolander Jr JE, Saito S. Fracture analyses using spring networks with random geometry. Engineering Fracture Mechanics 1998; 61:569-591.

11. Yang WH, Srolovitz DJ, Hassold GN, Anderson MP. Microstructural effects in the fracture of brittle materials. In Simulation and Theory of Evolving Microstructures, Anderson MP, Rollett AD (eds). The Metallurgical Society: Warrendale, PA, 1990, pp. 277-284.

12. Potts RB. Some generalized order-disorder transformations. Proceedings of the Cambridge Philosophical Society 1952; 48:106.

13. Srolovitz DJ, Anderson MP, Grest GS, Sahni PS. Grain growth in two dimensions. Scripta Metallurgica 1983; 17:241-246.

14. Srolovitz DJ, Anderson MP, Grest GS, Sahni PS. Computer simulation of grain growth: I. Kinetics. Acta Metallurgica 1984; 32(5):783-791.

15. Srolovitz DJ, Anderson MP, Sahni PS, Grest GS. Computer simulation of grain growth: II. Grain size distribution, topology and local dynamics. Acta Metallurgica 1984; 32(5):793-802.

16. Grest GS, Anderson MP, Srolovitz DJ. Computer simulation of normal grain growth in three dimensions. Philosophical Magazine 1988; B59(3):293-329.

17. Sridhar N, Yang W, Srolovitz DJ, Fuller Jr. ER. Microstructural mechanics model of anisotropic-thermalexpansion-induced microcracking. Journal of the American Ceramic Society 1994; 77(5):1123-1138.

18. Zimmermann A, Carter WC, Fuller Jr ER. Damage evolution during microcracking of brittle solids. Acta Materialia 2001; 49:127-137.

19. Holm EA. Surface formation energy for intergranular fracture in two-dimensional polycrystals. Journal of the American Ceramic Society 1998; 81(3):455-459.

20. Kim BN, Wakayama S, Kawahara M. Characteristics of 2-dimensional crack propagation behavior by simulation and analysis. International Journal of Fracture 1996; 75:247-259.

21. Zhai J, Zhou M. Finite element analysis of micromechanical failure modes in a heterogeneous ceramic material system. International Journal of Fracture 2000; 101:161-180.

22. Xu X-P, Needleman A. Numerical simulations of fast crack growth in brittle solids. Journal of the Mechanics and Physics of Solids 1994; 42:1397-1434.

23. Zavattieri PD, Raghuram PV, Espinosa HD. A computational model of ceramic microstructures subjected to multi-axial dynamic loading. Journal of the Mechanics and Physics of Solids 2001; 49:27-68.

24. Klein PA, Foulk JW, Chen EP. Numerical artifacts in cohesive fracture simulation. In Advances in Computational Engineering and Sciences. Atluri SN, Brust FW (eds). International Conferences on Computational Engineering and Sciences, 2000, pp. 1790-1795. 
25. Ortiz M, Leroy Y, Needleman A. A finite element method for localized failure analysis. Computer Methods in Applied Mechanics and Engineering 1987; 61:189-214.

26. Belytschko T, Fish J, Engelmann BE. A finite element with embedded localization zones. Computer Methods in Applied Mechanics and Engineering 1988; 70:59-89.

27. Dvorkin EN, Cuitiño AM, Gioia G. Finite elements with displacement interpolated embedded localization lines insensitive to mesh size and distortions. International Journal for Numerical Methods in Engineering 1990; 30:541-564.

28. Simo JC, Oliver J, Armero F. An analysis of strong discontinuities induced by strain softening in rateindependent inelastics solids. Computational Mechanics 1993; 12:277-296.

29. Simo JC, Oliver J. Modelling strong discontinuities in solid mechanics by means of strain softening constitutive equations. In Computational Modelling of Concrete Structures, Mang H, Bićanić N, de Borst R (eds). Pineridge: Swansea, 1994, pp. 363-372.

30. Armero F, Garikipati K. An analysis of strong discontinuities in multiplicative finite strain plasticity and their relation with the numerical simulation of strain localization in solids. International Journal of Solids and Structures 1996; 33:2863-2885.

31. Sluys LJ, Berends AH. Discontinuous failure analysis for mode-I and mode-II localization problems. International Journal of Solids and Structures 1998; 35:4257-4274.

32. Larsson R, Steinmann P, Runesson K. Finite element embedded localization band for finite strain plasticity based on a regularized strong discontinuity. Mechanics of Cohesive-frictional Materials 1999; 4(2):171-194.

33. Regueiro RA, Borja RI. Plane strain finite element analysis of pressure sensitive plasticity with strong discontinuity. International Journal of Solids and Structures 2001; 38(21):3647-3672.

34. Jirásek M. Comparative study on finite elements with embedded discontinuities. Computer Methods in Applied Mechanics and Engineering 2000; 188:307-330.

35. Moës N, Dolbow J, Belytschko T. A finite element method for crack growth without remeshing. International Journal for Numerical Methods in Engineering 1999; 46(1):131-150.

36. Daux C, Moës N, Dolbow J, Sukumar N, Belytschko T. Arbitrary cracks and holes with the extended finite element method. International Journal for Numerical Methods in Engineering 2000; 48(12):1741-1760.

37. Belytschko T, Moës N, Usui S, Parimi C. Arbitrary discontinuities in finite elements. International Journal for Numerical Methods in Engineering 2001; 50(4):993-1013.

38. Melenk JM, Babuška I. The partition of unity finite element method: Basic theory and applications. Computer Methods in Applied Mechanics and Engineering 1996; 139:289-314.

39. Duarte CA, Oden JT. An $H-p$ adaptive method using clouds. Computer Methods in Applied Mechanics and Engineering 1996; 139:237-262.

40. Sukumar N, Moës N, Moran B, Belytschko T. Extended finite element method for three-dimensional crack modeling. International Journal for Numerical Methods in Engineering 2000; 48(11):1549-1570.

41. Oden JT, Duarte CA, Zienkiewicz OC. A new cloud-based $h p$ finite element method. Computer Methods in Applied Mechanics and Engineering 1998; 153(1-2):117-126.

42. Strouboulis T, Copps K, Babuška I. The generalized finite element method. Computer Methods in Applied Mechanics and Engineering 2001; 190(32-33):4081-4193.

43. Belytschko T, Black T. Elastic crack growth in finite elements with minimal remeshing. International Journal for Numerical Methods in Engineering 1999; 45(5):601-620.

44. Sukumar N, Chopp DL, Moran B. Extended finite element method and fast marching method for threedimensional fatigue crack propagation. Engineering Fracture Mechanics 2003; 70:29-48.

45. Chopp DL, Sukumar N. Fatigue crack propagation of multiple coplanar cracks with the coupled extended finite element method/fast marching method. International Journal of Engineering Science, 2003, in press.

46. Moës N, Gravouil A, Belytschko T. Non-planar 3D crack growth by the extended finite element and level sets. Part I: Mechanical model. International Journal for Numerical Methods in Engineering 2002; 53(11): 2549-2568.

47. Gravouil A, Moës N, Belytschko T. Non-planar 3D crack growth by the extended finite element and the level sets-Part II: Level set update. International Journal for Numerical Methods in Engineering 2002; 53(11):2569-2586.

48. Wells GN, Sluys LJ. A new method for modelling cohesive cracks using finite elements. International Journal for Numerical Methods in Engineering 2001; 50(12):2667-2682.

49. Sukumar N, Chopp DL, Moës N, Belytschko T. Modeling holes and inclusions by level sets in the extended finite element method. Computer Methods in Applied Mechanics and Engineering 2001; 190(46-47): 6183-6200.

50. Wu FY. The Potts model. Reviews of Modern Physics, 1982; 54:235-268.

51. Carter WC, Langer SA, Fuller Jr ER. Object-Oriented Finite Element Analysis (OOF). Available at http://www.ctcms.nist.gov/oof, National Institute for Standards and Technology, Gaithersburg, MD, 1998.

52. Langer SA, Fuller Jr ER, Carter WC. OOF: An image-based finite-element analysis of material microstructures. Computing in Science and Engineering 2001; 3(3):15-23. 
53. Ghosh S, Mukhopadhyay SN. A material based finite-element analysis of heterogeneous media involving Dirichlet tessellations. Computer Methods in Applied Mechanics and Engineering 1993; 104(2):211-247.

54. Ghosh S, Nowak Z, Lee K. Quantitative characterization and modeling of composite microstructures by Voronoi cells. Acta Materialia 1997; 45(6):2215-2234.

55. Ghosh S, Moorthy S. Elastic-plastic analysis of arbitrary heterogeneous materials with the Voronoi cell finiteelement method. Computer Methods in Applied Mechanics and Engineering 1995; 121(1-4):373-409.

56. Gottstein G, Shvindlerman LS. Grain Boundary Migration in Metals: Thermodynamics, Kinetics, Applications. CRC Press: Boca Raton, FL, 1999.

57. Lee DT, Lin AK. Generalized Delaunay triangulation for planar graphs. Discrete and Computational Geometry 1986; 1:201-217.

58. Tanemura M, Ogawa T, Ogita W. A new algorithm for three-dimensional Voronoi tesselation. Journal of Computational Physics 1983; 51:191-207.

59. Bowyer A. Computing Dirichlet tessellations. The Computer Journal 1981; 24:162-166.

60. Watson DF. Computing the $n$-dimensional Delaunay tessellation with application to Voronoi polytopes. The Computer Journal 1981; 24(2):167-172.

61. Hassan O, Bayne LB, Morgan K, Weatherill NP. An adaptive unstructured mesh method for transient flows involving moving boundaries. In European Congress on Computational Methods in Applied Sciences and Engineering, ECCOMAS 1998, Barcelona, Spain, September 1998; 668-674.

62. George PL, Borouchaki H. Delaunay Triangulation and Meshing. Hermes Science Publications: Paris, France, 1998.

63. Holmes DG, Snyder DD. The generation of unstructured triangular meshes using Delaunay triangulations. In Proceedings of the 2nd International Conference on Numerical Grid Generation, Swansea, UK, 1988. Pineridge Press: Swansea, pp. 643-652.

64. Rebay S. Efficient unstructured mesh generation by means of Delaunay triangulation and Bowyer-Watson algorithm. Journal of Computational Physics 1993; 106:125-138.

65. Chew LP. Guaranteed-quality mesh generation for curved surfaces. In Proceedings of the 9th Annual ACM Symposium on Computational Geometry 1993; 274-280.

66. Baker TJ. Triangulations, mesh generation and point placement strategies. In Frontiers of Computational Fluid Dynamics, Caughey DA, Hafez MM (eds). Wiley: New York, 1994, pp. 101-115.

67. Moran B, Shih CF. Crack tip and associated domain integrals from momentum and energy balance. Engineering Fracture Mechanics 1987; 27(6):615-641.

68. Nikishkov GP, Atluri SN. Calculation of fracture mechanics parameters for an arbitrary three-dimensional crack by the 'equivalent domain integral method'. International Journal for Numerical Methods in Engineering 1987; 24:1801-1821.

69. Yau JF, Wang SS, Corten HT. A mixed-mode crack analysis of isotropic solids using conservation laws of elasticity. Journal of Applied Mechanics (ASME) 1980; 47:335-341.

70. Erdogan F, Sih GC. On the crack extension in plates under plane loading and transverse shear. Journal of Basic Engineering 1963; 85:519-527. 